\title{
LAMINITIS AND SOLE LESIONS IN DAIRY COWS; PATHOGENESIS, RISK FACTORS, AND PRECAUTIONS
}

\author{
Christer Bergsten
}

Swedish Dairy Association, PO BOX 234, Skara Sweden

Healthy feet and legs are of most concern for dairy cows well-being and performance. Lameness is a serious consequence of feet and leg disorders especially in high yielding cows as production and fertility are often affected. With larger and more confined dairy systems, claw disorders may be less often detected at an early stage and thus more difficult to treat. In a UK study the mean yearly treatment incidence risk of lameness was $55 \%$ and in a Minnesota study the prevalence of lameness was nearly $20 \%$. The most common claw lesions resulting in lameness were sole ulcer, sole abscess, digital dermatitis and foot rot. There are several sole lesions associated with laminitis such as sole and white line haemorrhages, fissures of the white line, and double soles. These lesions can be observed even if lameness is not evident and they can develop to more severe lesions such as sole ulcers and sole abscesses. Sole lesions are used in the research to identify risk factors for lameness and laminitis with the aim to promote claw health.

Acute laminitis is less common in dairy cows while subacute and subclinical laminitis results in the most common claw lesions in today's dairy production. Researchers believe that the inflammation in laminitis is primarily caused by a circulatory disturbance in the feet that results in hypoxia and malnutrition of the corium connecting the claw bone to the wall. Due to mechanical stretching, the claw bone can rotate and or sink inside the claw. Depending on the severity of the initial lesion, the mobility of the claw bone inside the capsule and the counter pressure on the sole from hard floors and uneven loading, the sole corium can suffer contusions and secondary lesions of the sole area can develop. The lesions on the sole can be looked upon as a print of the bone where it hits the sole. Sole ulcer typically shows up at the position corresponding to the "heel" of the claw bone.

Laminitis with secondary claw lesions is often a herd problem. As with other production diseases, laminitis is a multifactorial disease and management decisions are critical for it's development. It has been disputed in what extent laminitis and sole lesions are influenced by feeding and trauma per se. In a series of metabolic studies, lower fiber content, higher concentrate-forage ratio, higher concentrate amount, and higher dietary protein intake, all resulted in higher lameness scores, associated with sole lesions, than in controls fed less intensive diets. Also the feeding management and roughage quality are important risk factor for laminitis. It is no doubt from several studies that the hardness of the floors is of crucial importance for the development of sole lesions. Rubber mats thus reduced the sole lesions in comparison to concrete floors. Uncomfortable cubicles and over crowding have showed to increase sole lesions due to too much standing. Cows must be allowed to lie down and relieve soar feet from loading. Both metabolic and traumatic changes occur around calving in dairy cows, which make this period critical.

It is important for laminitis precautions to reduce sudden changes of feeding and environment during the calving period and that the animals, especially first calving heifers, get accustomed to the dairy cows facilities smoothly. Claw trimming has proved to reduce lameness associated with laminitis because load is more equally distributed and subclinical lesions can be observed before they get complicated. 